\title{
Laparoscopic antireflux surgery - Our initial experience
}

Pradhan S, Bikal Ghimire, Kansakar P, Y P Singh, P Vaidya, P Sayami

Department of Surgery,

Tribhuvan University Teaching Hospital, Kathmandu

Correspondence: Dr Sumita Pradhan ,MCh resident Surgical Gastroenterology, Department of Surgery, Tribhuvan University Teaching Hospital, Phone No: 9841198822

Email: sumiepradhan@gmail.com

\begin{abstract}
Introduction: Laparoscopic antireflux surgery (LARS) currently represents the gold standard in the surgical management of gastrointestinal reflux disease (GERD) with minimal morbidity and mortality. Routine fundoplication following laparoscopic Heller's cardiomyotomy is also being recommended to reduce the incidence of pathological gastro-oesophageal reflux after surgery. The aim of the current study was to evaluate patients receiving LARS and to assess their surgical outcomes
\end{abstract}

Methods: Prospective data of all patients admitted in our department with these diseases and undergoing LARS, from May 2014 to November 2015 were reviewed. Patients with Achalasia cardia underwent Laparoscopic Heller's cardiomyotomy with Dor's fundoplication and those with GERD with hiatus hernia underwent Laparoscopic Toupet's fundoplication. Age, sex, duration of surgery, surgical morbidity and hospital stay were recorded.

Results: Eleven patients underwent LARS. Females were 5(45.5\%) and males were 6 (54.5\%). Mean age of patients was $36.18 \pm 15.79$ years (range 18-68 years). 6 patients (54.5\%) underwent Laparoscopic Heller's cardiomyotomy with Dor's fundoplication for Achalasia cardia while 5 patients (45.5\%) underwent Laparoscopic fundoplication. The median operating room time was $133.64 \pm 15.66$ minutes (range, 110-160). There were no conversions. The median hospital stay was $3.45 \pm 0.522$ days (range, 3-4 days). No postoperative complications or preoperative deaths occurred. No patient had a perforation revealed on the postoperative contrast swallow when performed. Gastro esophageal reflux symptoms were significantly improved and severity of dysphagia was also reduced after surgery. The average follow-up period is $5.45 \pm 2.67$ months (range, 3- 12).

Conclusion: LARS is well established technique and becoming more popular over conventional open surgery in view of its equal safety and efficacy with added advantage of less morbidity and mortality. However larger case series and long term follow up would be warranted.

Key Words: Laparoscopic antireflux surgery, Hiatus hernia, GERD, Achalasia cardia 